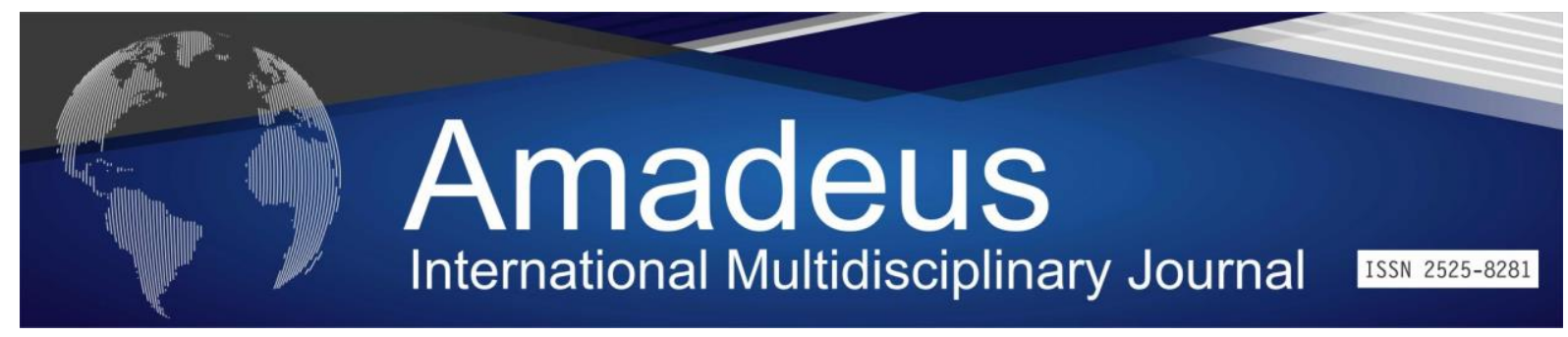

DOI: 10.14295/aimj.v5i10.156

\title{
Right Diaphragmatic Hernia: A Case Report
}

Igor de Morais Marques ${ }^{1}$, Ana Lígia Rocha Peixoto ${ }^{2}$, Marcelo Félix de Freitas ${ }^{3}$, Luis Henrique Santana Grangeiro $^{4}$, Francisco Felippe de Araújo Rolim ${ }^{5}$

Eleazar Menezes Araujo ${ }^{6}$, Maria Livia de Morais Marques $^{7}$
Abstract: Case report of a patient with chronic right diaphragmatic hernia. Patient in the emergency room of a trauma referral hospital with severe intestinal obstruction. Posttraumatic diaphragmatic hernia is an injury rarely found in medical practice, especially when it affects the right hemidiaphragm. The ruptures on the right side are associated with high morbidity and mortality and, therefore, the underdiagnosis of injuries on the right may be due to higher pre-hospital mortality or to a delay in the inhospital diagnosis, often because of the variable degree of traumatic involvement. Blunt diaphragmatic rupture, as it is an uncommon condition, should have a high index of suspicion in patients suffering from thoracoabdominal trauma, regardless of the trauma mechanism. The association with multiple and severe injuries is frequent, presenting a considerable morbidity and mortality rate, due to the risks of immediate and late complications involved in the delay in diagnosis.

Keywords: Diaphragmatic hernia; right hemidiaphragm; diagnosis.

\section{Hérnia Diafragmática à Direita: Um Relato de Caso}

\begin{abstract}
Resumo: Relato de caso de um paciente com hérnia diafragmática direita crônica. Paciente em emergência de hospital referência de trauma com obstrução intestinal grave. Hérnia diafragmática pós-traumática é uma injúria pouco encontrada na prática médica, principalmente quando acomete o hemidiafragma direito. As rupturas do lado direito estão associadas a alta morbi-mortalidade e, portanto, o subdiagnóstico de lesões à direita pode ser devido a uma maior mortalidade pré-hospitalar ou a um atraso no diagnóstico intra-hospitalar, muitas vezes em consequência do grau variável de acometimento traumático. A ruptura diafragmática contusa, por ser uma condição pouco frequente, deve ter alto índice de suspeição nos pacientes vítima de trauma tóraco-abdominal, independente do mecanismo de trauma. A associação com lesões múltiplas e graves é frequente, apresentando considerável taxa de morbi-mortalidade, devido aos riscos de complicações imediatas e tardias envolvidas no atraso diagnóstico.
\end{abstract}

Palavras-chave: Hérnia diafragmática; hemidiafragma direito; diagnóstico. 
${ }^{2}$ Coloproctologista/ Hospital Regional do Cariri. analigiarocha@ yahoo.com.br

${ }^{3}$ Cirurgião geral/ Hospital Regional do Cariri. marcelofelixdefreitas@yahoo.com.br

${ }^{4}$ Cirurgião Oncológico/ Hospital Regional do Cariri. lhsgrangeiro@ hotmail.com

${ }^{5}$ Cirurgião do Aparelho Digestivo/ Hospital Regional do Cariri. felippe.rolim1@gmail.com

${ }^{6}$ Urologista/Hospital Regional do Cariri. eleazar.araujo@yahoo.com.br

${ }^{7}$ Acadêmica de medicina/FAMENE-Mossoró. liviadmmarques@hotmail.com

\section{Introdução}

Hérnia diafragmática pós-traumática é uma injúria pouco encontrada na prática médica, principalmente quando acomete o hemidiafragma direito. A ruptura traumática do diafragma pode ocorrer em razão de lesões penetrantes e em decorrência de trauma contuso grave, com incidência de $10-15 \%$ e de 1-7\%, respectivamente.[1] São mais diagnosticadas no lado esquerdo, pois o fígado oblitera o defeito ou o protege no lado direito.

A incidência de hérnia diafragmática traumática é de 0,8-1,6\% em pacientes internados em hospital por trauma contuso, sendo responsável por $75 \%$ das rupturas, e apresentando uma proporção homem/mulher de 4:1.[2] Órgãos abdominais podem herniar imediatamente, através do defeito no diafragma, na cavidade pleural ou podem, gradualmente, penetrar no tórax em meses a anos. Carter et al. [3] observou que os pacientes podem experimentar um período assintomático, conhecido como "fase latente", por décadas antes de finalmente manifestar sintomas, às vezes com complicações graves.

As rupturas do lado direito estão associadas a alta morbi-mortalidade e, portanto, o subdiagnóstico de lesões à direita pode ser devido a uma maior mortalidade pré-hospitalar ou a um atraso no diagnóstico intra-hospitalar, muitas vezes em consequência do grau variável de acometimento traumático. O método de imagem mais disponível nos hospitais é a radiografia de tórax, com sensibilidade de $46 \%$ para rupturas do lado esquerdo e de $17 \%$ no lado direito. Nos centros em que há a disponibilidade de tomografia computadorizada a sensibilidade aumenta para $73 \%$ e a especificidade para 90\%.[4] Outras modalidades diagnósticas incluem a ultrassonagrafia, ressonância magnética, cintilografia de fígado/baço, laparoscopia e toracoscopia.

Para correção, o reparo das hernias é invariavelmente cirúrgico. Nos casos de ruptura aguda, a laparotomia é o método de primeira escolha no acesso à lesão diafragmática. Já 
em casos mais tardios, toracotomia ou laparotomia deve ser realizado, muito embora tanto a laparoscopia quanto a toracoscopia tenham sido relatadas na literatura como ferramentas de reparo de lesões diafragmáticas traumáticas.[5]

\section{Relato de Caso}

Homem de 46 anos, sem comorbidades, ajudante de pedreiro, foi admitido na emergência com história de dor abdominal em região epigástrica e constipação há 5 dias. Evoluiu com intensificação dos sintomas e associação a vômitos, disfagia, dor torácica e dispnéia há 3 dias. Nega cirurgias prévias ou internamentos anteriores. Relata episódio de queda de andaime de aproximadamente 3 metros de altura há 3 anos com fratura de arco costal à direita, porém tratado conservadoramente.

Ao exame físico, apresenta-se orientado, cooperativo, taquidispnéico em uso de suporte de oxigênio com satO2 de 90\%, taquicárdico, temperatura de 35o, sudoréico e hipocorado; ausculta cardíaca com bulhas hipofonéticas, diminuição dos murmúrios vesiculares no hemitórax direito, e abdome bastante distendido com dor à palpação em hipocôndrio direito. Exames complementares: hemoglobina 15,4g/dl, hematócrito de 47\%, leucócitos 10 400, plaquetas 272 000, inr 1,6 segundos, lactato 5,8, ph 7,44, paO2 $88 \mathrm{mmHg}, \mathrm{pCO} 241,9 \mathrm{mmHg}, \mathrm{HCO} 328,5 \mathrm{mEq} / \mathrm{l}$, $\mathrm{FiO} 250 \%$ troponinas negativas. Tomografia total de abdome sem contraste evidenciou hérnia diafragmática direita com insinuação de alças colônicas determinando processo obstrutivo intestinal a montante e compressão das estruturas mediastinais; deslocamento contralateral hepático secundário a compressão extrínseca intestinal. Tomografia computadorizada do tórax evidenciava atelectasia pulmonar compressivo total direita e faixas atelectásicas à esquerda; derrame pleural à direita com volume estimado em $360 \mathrm{ml}$. Realizada intubação orotraqueal e iniciado reposição volêmica.

O paciente foi submetido a laparotomia mediana xifopubiana. $\mathrm{Na}$ inspeção da cavidade, um defeito aproximado de $5 \mathrm{~cm}$ de diâmetro foi visualizado na hemicúpula diafragmática direita, com herniação do ceco, cólon ascendente e transverso, e distenção importante de alças de delgado na cavidade abdomial. Foi realizado frenotomia com liberação ampla de anel diafragmático por dificuldade de redução da hérnia; colectomia 
direita e de transverso com íleo cólon anastomose término-lateral devido à presença de áreas de necrose do cólon em conteúdo herniário. O defeito foi reparado com sutura direta, utilizando-se fio inabsorvível. O tórax à direita também foi drenado. Após 9 dias de pósoperatório sem intercorrências, o paciente recebeu alta hospitalar.

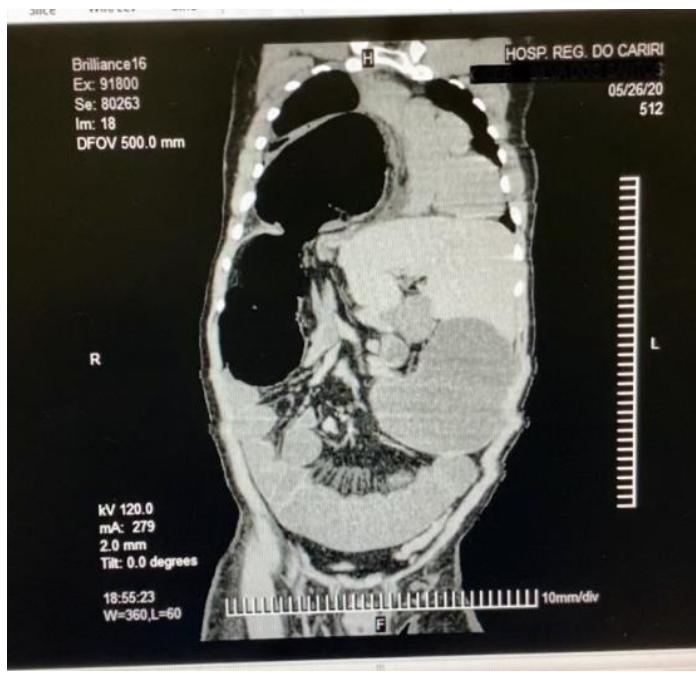

Figura 1: Tc em corte coronal demonstrando insinuação de alças colônicas em cavidade torácica

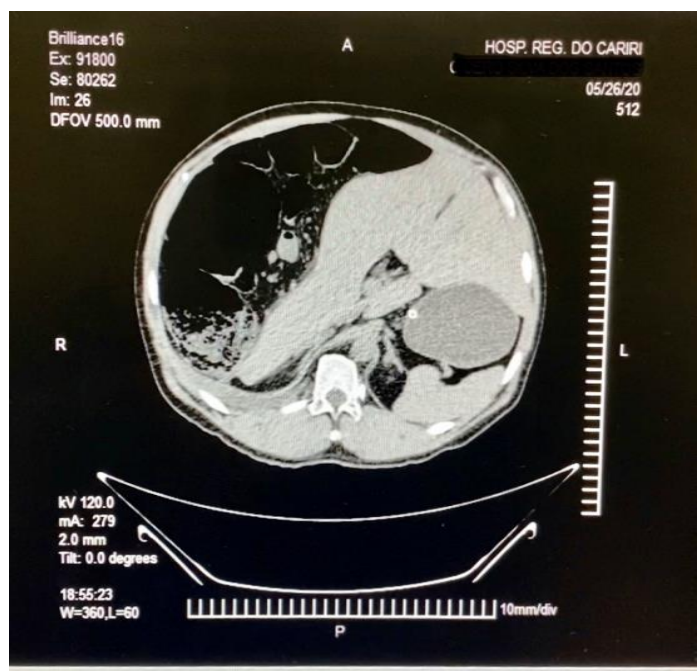

Figura 2: Tc em corte axial demonstrando alças cólicas deslocando o fígado medialmente 


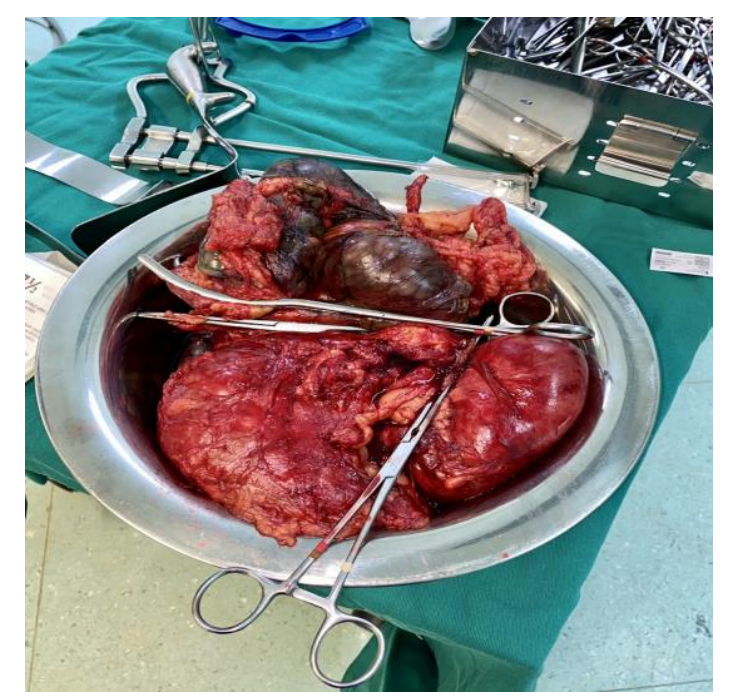

Figura 3: Conteúdo intra-abdominal removido da cavidade torácica após a cirurgia

\section{Discussão}

A hérnia diafragmática ocorre em decorrência de um defeito nessa musculatura, permitindo com que órgãos abdominais penetrem na cavidade torácica. Estes defeitos podem ser adquiridos ou congênitos.

A hérnia diafragmática adquirida resulta, na maioria dos casos, em decorrência de trauma tóraco-abdominal contuso ou penetrante. O trauma contuso produz grandes lacerações radiais que levam à hérnia, enquanto o trauma penetrante produz pequenas perfurações que podem permanecer assintomáticas durante meses a anos.

Grimes[6] descreveu três fases clínicas das lesões diafragmáticas: 1)fase aguda, onde a lesão é adequadamente identificada na primeira avaliação do paciente com reparo cirúrgico imediato; 2)fase intermediária ou latente, quando o diagnóstico passa despercebido na fase aguda. Apresenta sintomas vagos mas que podem evoluir com a progressão do encarceramento causando dispnéia, dor epigástrica e sintomas de obstrução intestinal; 3)fase crônica, que caracteriza-se pela presença das complicações, principalmente obstrução e estrangulamento intestinal. 
Os principais sinais e sintomas incluem a diminuição do murmúrio vesicular (64,2\%), dispnéia (42,8\%), ruídos hidro-aéreos auscultáveis no tórax (35,7\%), náuseas e vômitos (35,7\%) e dor abdominal (28,5\%).[7] Nos pacientes na fase crônica, as queixas gastrointestinais causadas pelo encarceramento das vísceras intra-abdominais são mais comuns que os sintomas respiratórios (7:1), que são mais prevalentes na fase aguda.[8] Os órgãos típicos que herniam para a cavidade torácica incluem estômago, baço, cólon, intestino delgado e fígado. Gaine F.A. et al[2], em 2013, mostrou que os conteúdos herniais mais comuns foram omento e estômago, presentes em 87,9\% e 69,7\%, respectivamente, seguidos de intestino delgado $33,3 \%$, intestino grosso $28,6 \%$ e baço em 9,5\%. Dentre as complicações mais comuns, destacam-se a hemorragia e a obstrução, que podem evoluir com insuficiência cardiorrespiratória progressiva, instabilidade hemodinâmica grave e isquemia de vísceras.

O diagnóstico é de difícil suspeição, pois, geralmente, o quadro clínico é assintomático ou a sua apresentação é dominada por outros sintomas de lesões associadas. A radiografia de tórax ajuda no diagnóstico mostrando com maior frequência as seguintes anormalidades: elevação e irregularidade do hemidiafragma acometido, padrão anormal de gás acima do diafragma com alças distendidas e a posição do cateter nasogástrico dentro do hemitórax (método simples e útil na identificação da câmara gástrica), principalmente em casos de trauma contuso.[9]

Estudos recentes mostram que a tomografia computadorizada helicoidal e multislice pode ser considerada o padrão-ouro para o diagnóstico, permitindo uma melhor demonstração dos sinais mais sutis de lesões diafragmáticas com maior sensibilidade, especificidade e precisão.[10] Os achados sugestivos de hérnia direita vistos à TC são espessamento do diafragma, corcova e sinais de herniação de parte do fígado.[11] A ressonância magnética também é útil, mas geralmente não é realizada em situações de emergência, no entanto, pode ser empregada em pacientes estáveis ou quando a TC é ambígua.

O tratamento cirúrgico é mandatório sempre que for feito o diagnóstico, pois não há qualquer possibilidade de fechamento espontâneo deste tipo de lesão e a diferença que ocorre entre o gradiente de pressão no abdome e no tórax favorece o aumento da herniação. O reparo cirúrgico da ruptura diafragmática é curativo, suportando bem as suturas e com baixíssima taxa de recorrência. Este pode ser realizado por meio de sutura primária ou com 
o uso de tela. Embora o tipo de fechamento ainda seja motivo de debate, é aceito que a maioria dos defeitos podem ser primariamente fechados por sutura não absorvível.

A laparoscopia é muito útil tanto para tratamento quanto para diagnóstico. Uma revisão sistemática recente que incluiu 2023 pacientes mostrou que, para hérnia diafragmática traumática aguda, uma abordagem abdominal é mais frequente que uma torácica, enquanto que na fase crônica, a via torácica é mais comum.[12] Um estudo americano, em 2012, demonstrou que a maioria dos reparos é realizado utilizando técnicas abdominais e torácicas abertas, com baixa mortalidade operatória e sem diferença significativa entre a cirurgia aberta ou por vídeo. Não houve diferença entre as taxas de pneumonia pós-operatória, tvp, iam ou sepse entre os tipos de abordagens, no entanto, os reparos laparoscópicos estão associados a menor tempo de internação.[13] A escolha da abordagem cirúrgica difere bastante na literatura e depende em grande parte da preferência e aptidão do cirurgião ou da prática exercida no serviço de origem.

\section{Considerações finais}

A ruptura diafragmática contusa, por ser uma condição pouco frequente, deve ter alto índice de suspeição nos pacientes vítima de trauma tóraco-abdominal, independente do mecanismo de trauma. A associação com lesões múltiplas e graves é frequente, apresentando considerável taxa de morbi-mortalidade, devido aos riscos de complicações imediatas e tardias envolvidas no atraso diagnóstico. O diagnóstico pode ser feito clinicamente ou por exames de imagem, podendo utilizar laparoscopia/toracoscopia quando não for possível excluir a ruptura por métodos não invasivos. O tratamento da lesão é eminentemente cirúrgico, sobretudo em situações de emergência.

\section{Referências}

[1] Lee J.Y., Sul Y.H., Ye J.B., Ko S.J., Choi J.H., Kim J.S., Right-sided diaphragmatic rupture in a poly traumatized patient. Annals of surgical treatmente and reserch, 2017 august. 
[2] Gaine F.A., Lone G.N., Chowdhary M.A., Lone H., The etiology, associated injuries and clinical presentation of post traumatic of diaphragmatic hernia. Bulletin of emergency and trauma, 2013 1(2): 76-80

[3] Carter B.N., Giuseffi J., Felson B., Traumatic diaphragmatic hernia. Am J Roentgenol Radium Ther. 1951;65(1):56-72. Pubmed.

[4] Rashid F., Chakrabarty M.M., Singh R., Iftikhar S.Y., A review on delayed presentation of diaphragmatic rupture. Wourld journal of emergency surgery, 2009 august

[5] Matthews B.D., Bui H., Harold K.L, Kercher K.W., Andrales G., Park A., Sing R.F., Heniford B.T., Laparoscopic repair of traumatic diaphragmatic injuries. Surg endoscop. $2003 \mathrm{feb} ; 17(2): 254-8$

[6] Grimes O., Traumatic injuries of the diaphragm. American Journal Surg 1974; 128: $175-81$.

[7] Parreira J.M., Chibata M., Skinovsky J., Saucedo-junior N., Martins A.M., Schmidt F.R., Hérnia diafragmática direita tardia associada a hepatotórax - relato de caso com revisão da literatura. Arquivo brasileiro de cirurgia digestiva, 2009; 22(3): 183-5

[8] Gu P., Lu Y., Li X., Lin X., Acute and chronic traumatic diaphragmatic hernia: 10 years' experience. Plos one, 2019 december

[9] Pereira junior G.A., Hérnia diafragmática traumática. Revista do colégio brasileiro de cirurgiões, outubro de 2000; 375-382

[10] Dwivedi S., Banode P., Gharde P., Bhatt M., Ratanlal Johrapurkar S. Treating traumatic injuries of the diaphragm. Journal Emergency Trauma Shock 2010; 3: 173-6.

[11] Ties J.S., Peschman J.R., Moreno A., Mathiason M.A., Kallies K.J., Martin R.F., et al. Evolution in the management of traumatic diaphragmatic injuries: a multi-center review. Journal Trauma Acute Care Surgery 2014; 76: 1024-8. 
[12] Silva G.P., Cataneo D.C., Cataneo A.J.M. Thoracotomy compared to laparotomy in the traumatic diaphragmatic hernia. Systematic review and proportional methanalysis. Acta Cir Bras. 2018; 33(1): 49-66.

[13] Paul S., Nasar A., Port J.L., Lee P.C., Stiles B.C., Nguyen A.B., Altorki N.K., Sedrakyan A. Comparative analysis of diaphragmatic hernia repair outcomes using the nationwide inpatient sample database. Arch Surg. 2012, 147: 607-612.

[14] Doherty G.M., Current diagnóstico e tratamento, 14 edição. Editora artmed. 2017, 1101-1102.

\section{How to cite this article (APA format):}

Marques, Igor de Morais; Peixoto, Ana Ligia Rocha; Freitas, Marcelo Félix de; Grangeiro, Luis Henrique Santana; Rolim, Francisco Felippe de Araújo; Araujo, Eleazar Menezes; Marques, Maria Livia de Morais. (2020). Right Diaphragmatic Hernia: A Case Report. Am. In. Mult. J., Mar. to Jun. (10) 5, 7-15.

Received: 03/19/2021

Accepted: 03/26/2021 